\title{
Learning the Causal Graph of Markov Time Series
}

\author{
Avhishek Chatterjee, Ankit Singh Rawat, Sriram Vishwanath and Sujay Sanghavi \\ Electrical and Computer Engineering \\ The University of Texas at Austin \\ Austin, Texas \\ Email:avhishek,ankitsr@utexas.edu, sriram@ece.utexas.edu, sanghavi@mail.utexas.edu
}

\begin{abstract}
This paper considers a natural and widely prevalent setting where a collection of one dimensional time series evolve in a causal manner, and one is interested in inferring the graph governing the causality between these processes in a high dimensional setting. We consider this problem in the special case where variables are discrete and updates are Markov. We develop a new algorithm to learn causal graph structure based on the notion of directed information, and analytically and empirically demonstrate its performance. Our algorithm is an adaptation of a greedy heuristic for learning undirected graphical models, with modifications to leverage causality. Analytically, the challenge lies in determining sample complexity, given the dependencies between samples.
\end{abstract}

\section{INTRODUCTION}

In this paper, we investigate methodologies for learning the (Markov) graph that represents the dependencies between parameters in time series data. Specifically, we study the following problem: We are given a collection $\mathbf{X}_{v}, v \in \mathcal{V}$ of time series whose evolution is governed by a directed graph. For each $v \in \mathcal{V}$, the value $X_{v}^{t+1}$ at time $t+1$ depends on the values $X_{i}^{t}$ of the set of parents of $v$ in the directed graph at time $t$, and conditioned on these, $X_{v}^{t+1}$ is independent of all other nodes. The task we focus on is stated as follows: On observing the processes $\left\{x_{v}^{t}, v \in \mathcal{V}\right\}$ for some time horizon $t \leq n$, we desire to find the corresponding directed graph associated with the dependencies within these processes.

Learning causal relationships in discrete-valued multivariate (or multi-dimensional) time series is important in many areas including epidemiology, safety planning, genetics, climate modeling, neuro-sciences, planning recommendation driven business. In all these cases it is often important to understand the cause-effect relationships between different components of the data. For example, learning causal relationships between different kinds of epidemics helps to take pre-cautions where as understanding the causality between neural-spikes at different locations in the nervous system unveils the functioning of neural systems. There are many such examples where it is important to learn the causal graph that encodes the causal relationships between components of a time-series.

In this work, we aim to learn the underlying directed graph of causality of a multivariate process (time-series) from an observation of finite duration. As many processes in real-life can be characterized using a finite-windowed memory, we

\footnotetext{
${ }^{1}$ We like to thank NSF grants 1017525 and 0954059 , and the Army Research Office (ARO) for supporting this research.
}

consider stationary Markov processes to capture a broad class of systems. We propose a low complexity greedy algorithm that bases on the concept of directed mutual information / conditional entropy. We provide theoretical guarantees on the successful recovery of the underlying directed graph and prove that the sample complexity of the algorithm is logarithmic in the number of process variables. We also show results for the performance of our proposed algorithm on computer generated data and also show that our algorithm can learn the parent-child relationships even when there are possibly cyclic causations.

The remainder of this paper is organized as follows. Section I-A discusses the approaches towards learning causality in time-series and the relevant fields for applications of this problem. In Sec. II, we explain the problem at hand in more detail. Sec. III provides a brief introduction to relevant concepts and describes the closely related work in [1] for learning undirected graphical models from i.i.d data and points out the differences with this work. In Sec. IV, we present a greedy algorithm for learning causal dependence (directed) graph for Markovian time-series and present correctness results and sample complexity bounds for the algorithm. In Sec. VI, we present simulation results.

\section{A. Related Work}

Undirected and directed graphical models have found successful application in diverse fields for studying systems of inter-dependent variables. In scenarios where the true dependence among these variables is unknown, there has been significant work on learning them from i.i.d samples [2], [3], [4]; following seminal work in this field by Chow and Liu [5]. Note that there are two families of graphical models: undirected, primarily used to capture statistical dependencies between variables, and directed graphical models that go a step further in representing causal relationships between them. To date, a majority of existing literature in graphical models has focused on undirected ones, including those cited above. In recent years, the concept of graphical models has been extended to study inter-dependent time-series [6],[7]. In this context, the tail of an edge of the directed graph is an 'influencing' process and head is an 'influenced' process. This idea of generalizing graphical model to time series has been applied to multiple domains including finance [8], medicine [9], neuro-science [10],[11].

Understanding the causality in dynamics of a system is equivalent to learning the underlying directed graph. Note 
that in learning the graph of a process the learner is given correlated samples from a multi-dimensional process rather than i.i.d vector samples. [7] learns the causal or directed graph of a stationary multivariate Gaussian process using frequency domain methods. [12] proposes a method of polynomial sample complexity for learning graph of real-valued linear time-invariant systems.

Due to its many application in genetics, epidemiology, climate modeling and neuro-sciences, learning causality in discrete-valued (or non-numeric valued) time series is of immense importance [13], [14], [15], [10], [13]. Towards this insights obtained from the rich literature on learning graphical models from discrete valued samples are useful. In their seminal work, Chow and Liu learn a tree model for dependent random variables by running max-weight spanning tree algorithm with edge weight being the estimate of the mutual information between the variables computed from samples [5]. The information theoretic approach has been generalized to learn more general ensembles of undirected graphical models in [1], [16]. However as mutual information is symmetric function of the processes, it does not capture the direction of causation between them.

In [15], Kirshner et al. present a Chow-Liu type tree model for discrete-valued multivariate time series that captures dependencies across time and variables by representing each variable at each time by a node in a graph. Quinn et al. modify this model by representing each process as a node in a Chow-Liu type tree model where direction is associated with each edge to capture the causality between two processes [17].

Directed mutual information, a quantity first defined in [18], is an asymmetric quantity that captures dependencies between two processes and has been used to learn causality in systems (reviewed in [19]). In [17], Quinn et al. uses ChowLiu algorithm to learn directed tree for a stationary process using estimates of directed mutual information computed using universal source coding based method proposed in [20]. Though this method is asymptotically accurate when the true model is a tree, but has no guarantee on sample complexity. Moreover this method can not learn a general directed graph of causality.

A concurrent line of work studies time series using a dynamic Bayesian network approach as proposed in [21]. This idea has been extended and applied by [22], [23] and others. These papers address the case where causality structure is an acyclic graph and do not have theoretical guarantees on complete recovery of the underlying graph of causality.

\section{PROBlem SETting}

Consider a discrete-time multi-dimensional random process $\mathbf{X}=\left\{\mathbf{X}_{v}: v \in \mathcal{V}\right\}$ where $\mathcal{V}$ with $|\mathcal{V}|=p$ is the index-set for the one-dimensional component processes. The process is stationary and Markovian with the following transition probability kernel.

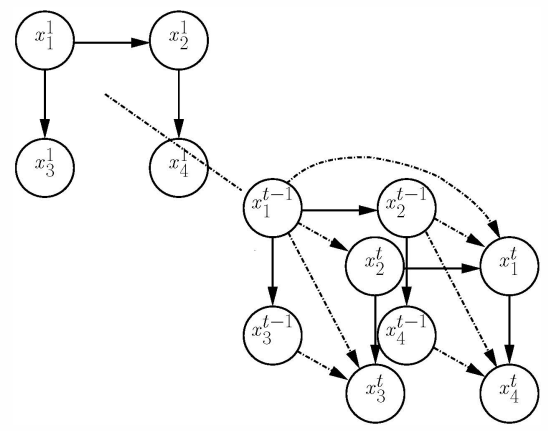

Fig. 1: Time evolution of a one-step Markov process without instantaneous effects. The dotted lines between the process values at $t$ and $t-1$ show the process influences. Solid lines between variables at time $t$ do not mean instantaneous effect, rather it is the abstract representation of causality structure (the cause and effect processes) across time that we want to learn.

$$
p\left(\mathrm{x}^{t} \mid \mathbf{x}^{t-1}\right)=\prod_{i \in \mathcal{V}} p\left(x_{i}^{t} \mid x_{i}^{t-1}, x_{\mathrm{pa}(i)}^{t-1}\right)
$$

Here $\mathrm{pa}(i)$ is the set of indices on which the component process $i$ depends. For an arbitrary Markovian probability transition kernel, these dependencies are not necessarily symmetric. Nevertheless, these dependencies can be represented by a graph, in this case one with directed edges where an edge from $i$ to $j$ implies that component process $j$ has Markovian dependence on the component process $i$.

In Figure 1 we present an example to illustrate the evolution of the process. $\mathbf{X}_{1}$ is a parent of $\mathbf{X}_{3}$, i.e. the present value of $\mathbf{X}_{3}$ is controlled by its own value and the value of $\mathbf{X}_{1}$ at the previous time step, i.e. given $X_{3}^{t-1}$ and $X_{1}^{t-1}, X_{3}^{t}$ does not depend on any other process values up to time $t$. Similar kind of dependence exists between $\mathbf{X}_{4}$ and $\mathbf{X}_{2}$. The dotted lines show this dependence among variables across time.

In this paper, we address the problem of learning the causal dependence among participating univariate time-series, given $n$ contiguous samples of $\mathbf{X}$. Therefore, our goal is to learn directed graph $\mathcal{G}$ associated with $\mathbf{X}$ from observations $\left\{\mathbf{X}^{t}, 1 \leq t \leq n\right\}$. Note that the samples of the process are not independent across time and learning the causal graph is not same as learning statistical similarity between component processes. Thus this problem involves a different challenge of learning propagation of influence from one component process to another using dependent samples.

\section{Preliminaries}

Given a variable $Y$ and a set of variables $Z_{B}$ taking values in a finite alphabet, conditional entropy of $Y$ given $Z_{B}$ is defined as $H\left(Y \mid Z_{B}\right)=\sum_{y, z_{\mathbf{B}}}-p\left(y, z_{B}\right) \log p\left(y \mid z_{B}\right)$. We always have $H(Y)=H(Y \mid \emptyset) \geq H\left(Y \mid Z_{B}\right)$ and the difference, $I\left(Y ; Z_{B}\right)=H(Y)-H\left(Y \mid Z_{B}\right)$, is called mutual information between $Y$ and $Z_{B}$. The conditional entropy 
$H\left(Y \mid Z_{B}\right)$ decreases with increasing dependence between $Y$ and $Z_{B}$, and is zero if and only if $Y$ is a function of $Z_{B}$, i.e., $Y$ is deterministically defined by $Z_{B}$ [24]. The well known local Markov property of an undirected graphical model can be stated in terms of conditional entropy as,

$$
H\left(X_{i} \mid X_{\mathcal{N}(i)}, X_{j}\right)=H\left(X_{i} \mid X_{\mathcal{N}(i)}\right) \text { for } j \notin \mathcal{N}(i)
$$

where $\mathcal{N}(i)$ is the neighborhood of node $i$ in the underlying graph. On the other hand for any node $j \in \mathcal{N}(i)$ and a set $A \subset \mathcal{V} \backslash\{i, j\}$, we have

$$
H\left(X_{i} \mid X_{A}\right)-H\left(X_{i} \mid X_{A}, X_{j}\right) \geq 0 .
$$

Based on this observation, Netrapalli et al. [1] propose a greedy iterative algorithm to learn an undirected graphical model from i.i.d. samples with empirical conditional entropy (entropy calculated using empirical probability measure) as a metric. The algorithm initializes the neighborhood estimate for each node $i$ as an empty set and at each iteration greedily includes that node into the neighborhood estimate, which reduces empirical conditional entropy of the node $i$ by maximum amount, when added to the current neighborhood estimate. The algorithm works well in practice and has provable guarantee for graphical models with correlation decay and large girths when (3) is true with the difference being uniformly bounded away from zero.

The aforementioned measures for dependence can be generalized for random processes. The dependence between process $\mathbf{X}_{i}=\left\{X_{i}^{t}, 1 \leq t \leq n\right\}$ and $\mathbf{X}_{j}=$ $\left\{X_{j}^{t}, 1 \leq t \leq n\right\}$ can be quantified by mutual information $I\left(X_{i}^{1: n} ; X_{j}^{1: n}\right)=\sum_{t=1}^{n} I\left(X_{i}^{1: n} ; X_{j}^{t} \mid X_{j}^{1: t-1}\right)$ or conditional entropy $H\left(\mathbf{X}_{i} \mid \mathbf{X}_{j}\right)=\sum_{t=1}^{n} H\left(X_{i}^{t} \mid X_{i}^{1: t-1}, X_{j}^{1: n}\right)$. However, both of these fail to capture the direction of causal effect between $\mathbf{X}_{i}$ and $\mathbf{X}_{j}$. Towards this, analogous information theoretic quantities namely directed mutual information and directed conditional entropy allow us to identify the direction of causal effect [18]. For a given set of process indices $B$, directed conditional entropy of $\mathbf{X}_{i}$ given $\mathbf{X}_{B}$ is defined as

$$
H\left(\mathbf{X}_{i}|| \mathbf{X}_{B}\right)=\sum_{t=1}^{n} H\left(X_{i}^{t} \mid X_{i}^{1: t-1}, X_{B}^{1: t-1}\right) .
$$

As conditioning reduces entropy [24], $H\left(\mathbf{X}_{i} \| \mathbf{X}_{B}\right) \leq$ $H\left(\mathbf{X}_{i} \| \mathbf{X}_{B^{\prime}}\right)$ for $B^{\prime} \subset B$. The smallest $B$ for which $H\left(\mathbf{X}_{i} \| \mathbf{X}_{B}\right)$ achieves minimum is $\mathrm{pa}(i)$. This follows from the property of the causality graph of a process (Sec. II) that implies $X_{i}^{t}$ is independent of $X_{k}^{1: t-1}$ given $X_{\mathrm{pa}(i)}^{1: t-1}$.

Under the one-step Markov assumption on $\mathbf{X}$ and by the spatial Markov property of the causality graph, for any $i \in \mathcal{V}$ and $B \supseteq \mathrm{pa}(i)$ we have

$$
\begin{aligned}
H\left(\mathbf{X}_{i} \| \mathbf{X}_{B}\right) & =\quad \sum_{t=1}^{n} H\left(X_{i}^{t} \mid X_{i}^{1: t-1}, X_{B}^{1: t-1}\right) \\
& =\quad \sum_{t=1}^{n} H\left(X_{i}^{t} \mid X_{i}^{t-1}, X_{B}^{t-1}\right)
\end{aligned}
$$

When $\mathbf{X}$ is stationary, we have

$$
\begin{aligned}
H\left(\mathbf{X}_{i}|| \mathbf{X}_{B}\right) & =H\left(X_{i}^{1}\right)+\sum_{t=2}^{n} H\left(X_{i}^{t} \mid X_{i}^{t-1}, X_{B}^{t-1}\right) \\
& =H\left(X_{i}^{1}\right)+(n-1) H\left(X_{i} \mid X_{i}^{-1}, X_{B}^{-1}\right)
\end{aligned}
$$

Here we drop the time index to denote each term by $H\left(X_{i} \mid X_{i}^{-1}, X_{B}^{-1}\right)$ where -1 in the superscript denotes a delay by one time step. Thus for sufficiently large $n$, obtaining the directed conditional entropy $H\left(\mathbf{X}_{i} \| \mathbf{X}_{B}\right)$ is same as obtaining $H\left(X_{i} \mid X_{i}^{-1}, X_{B}^{-1}\right)$.

Note that for node $i \in \mathcal{V}, A \subset \mathrm{pa}(i)$, and $j \in \operatorname{pa}(i) \backslash A$, we have

$$
H\left(X_{i} \mid X_{i}^{-1}, X_{A}^{-1}, X_{j}^{-1}\right) \leq H\left(X_{i} \mid X_{i}^{-1}, X_{A}^{-1}\right) .
$$

This follows from the fact that conditioning reduces entropy. Moreover, the spatial Markov property (eq. 1) ensures that we have

$$
H\left(X_{i} \mid X_{i}^{-1}, X_{\mathrm{pa}(i)}^{-1}\right) \leq H\left(X_{i} \mid X_{i}^{-1}, X_{B}^{-1}\right) \quad \forall B \subseteq \mathcal{V} .
$$

Based on (5), (6), and (7), we propose a greedy algorithm to learn causal dependency directed graph for $\mathbf{X}$ as described in Fig.3. Though $H\left(X_{i}, X_{i}^{-1}, X_{A}^{-1}\right)$ is the conditional directed entropy of $i$ given $A$ only if pa $(i) \subseteq A$, for ease of exposition with slight abuse of terminology we call it the conditional directed entropy of $i$ given $A$ for any $A$.

As we do not have the distribution, we compute empirical estimate of conditional entropies $\hat{H}(\cdot \cdot \cdot)$ using empirical probability distribution learnt from samples. Under the stationarity assumption on $\mathbf{X}$, empirical estimate for $p\left(X_{i}=x_{i}, X_{A}^{-1}=\right.$ $x_{A}$ ) can be obtained as follows,

$\hat{p}\left(\left(X_{i}, X_{A}^{-1}\right)=\left(x_{i}, x_{A}\right)\right)=\sum_{t=2}^{n} \frac{1\left(\left(X_{i}^{t},, X_{A}^{t-1}\right)=\left(x_{i}, x_{A}\right)\right)}{n-1}$

\section{Algorithm}

In this section we propose a low-complexity greedy algorithm to learn the underlying directed graph that encodes the causal relationships in a multi-dimensional time series. The algorithm uses conditional directed entropy introduced above as a metric for greedily constructing the graph of interest.

Idea: For $i, j \in \mathcal{V}$, if process $j$ causally influences process $i$, then directed entropy of the process $i$ conditioned on a set $A \not \ngtr j$ decreases when $j$ is included in the set $A$. Based on this observation we design the algorithm to estimate $\mathrm{pa}(i)$ iteratively. At each iteration it finds a node that reduces the conditional directed entropy of $i$ maximally, and includes it in the estimated set $\widehat{\mathrm{pa}}(i)$ if the reduction is non-negligible. In the end the algorithm prunes $\widehat{p a}$ to remove any superfluous inclusion.

The algorithm has two major parts, greedy and pruning. All greedy and pruning steps run for each node and can be run in parallel. Below we describe the main steps that the algorithm goes through for each node or process index $i$.

- Filtering: Removes process indices or nodes from the search that do not reduce directed entropy of process $i$ when conditioned on. 
- Greedy: This is the main part of the algorithm where the parent set $\widehat{\mathrm{pa}}(i)$ is estimated iteratively.

- Best: This step picks the process index $k$ that is best in terms of reducing conditional directed entropy of the process $i$ among the processes that have not been included in $\widehat{\mathrm{pa}}(i)$ yet (and also not a nonparent ancestor as ensured in the next step).

- Non-parent Ancestor: It checks whether conditional directed entropy of $i$ conditioned on the current $\widehat{\mathrm{pa}}(i)$ and $X_{k}^{-1}$ is less than the same conditioned on $\widehat{\mathrm{pa}}(i)$ and $X_{k}^{-2}$. A node $k$ that fails this test is included in the set of 'possible' non-parent ancestors Rej.

- If a chosen best node is not a non-parent ancestor as per the previous step, it is included into $\widehat{p a}(i)$. Once a new node is included into $\widehat{\text { pa }}(i)$, Rej is emptied to reconsider those nodes.

- Pruning: This step picks a node from the constructed $\widehat{\mathrm{pa}}(i)$ whose removal does not increase conditional directed entropy of $i$ given $\widehat{\mathrm{pa}}(i)$.

Filtering step reduces the search space and thus keeps the number of spurious inclusions small ensuring that the sample complexity of the pruning step is low.

In greedy steps, the algorithm finds a node that reduces the conditional directed entropy by maximal amount. Then it checks whether the influence of the 'best' node reaches $i$ in one time step or in multiple time steps. Note that an influential non-parent ancestor as in the diamond graph example in Figure 2a, may have stronger effect on $i$ than any of the parents. But the influence of $k$ reaches $i$ in more than one time step. This observation can be used to learn parents even in this diamond graph, which is a hard problem in case of learning undirected graphs [1].

On the other hand there may be a node $k$ that has a direct edge to a node $i$ in the causality graph and also has a different directed path to $i$. If the influence that reaches $i$ from $k$ on the other directed path is much stronger than the direct influence, then it may be included in the non-parent ancestor set at an iteration of the algorithm. Rej is emptied after every inclusion into pa $(i)$ to prevent such a node getting eliminated permanently.

The pruning step is important to avoid the corner cases like the one shown in Figure 2b. It may happen that an influential non-parent ancestor may effect another node $u$ very strongly. Thus in this way $u$ has indirect effect on $i$ and may be picked in the greedy step as 'best' node. Note that as effect from influential non-parent takes one time step to reach $u, u$ may pass the test for non-parent ancestor. To remove such superfluous inclusions pruning is important. Given the parents are already in $\widehat{\mathrm{pa}}(i)$, removal of any superfluous node does not increase conditional directed entropy of $i$. On the other hand, removal of any parent node increases conditional directed entropy of $i$ (section III).

For each node $i$ the algorithm iterates over all other nodes to pick the best node that minimizes conditional directed entropy. Even in extreme worst case at most $p$ such best

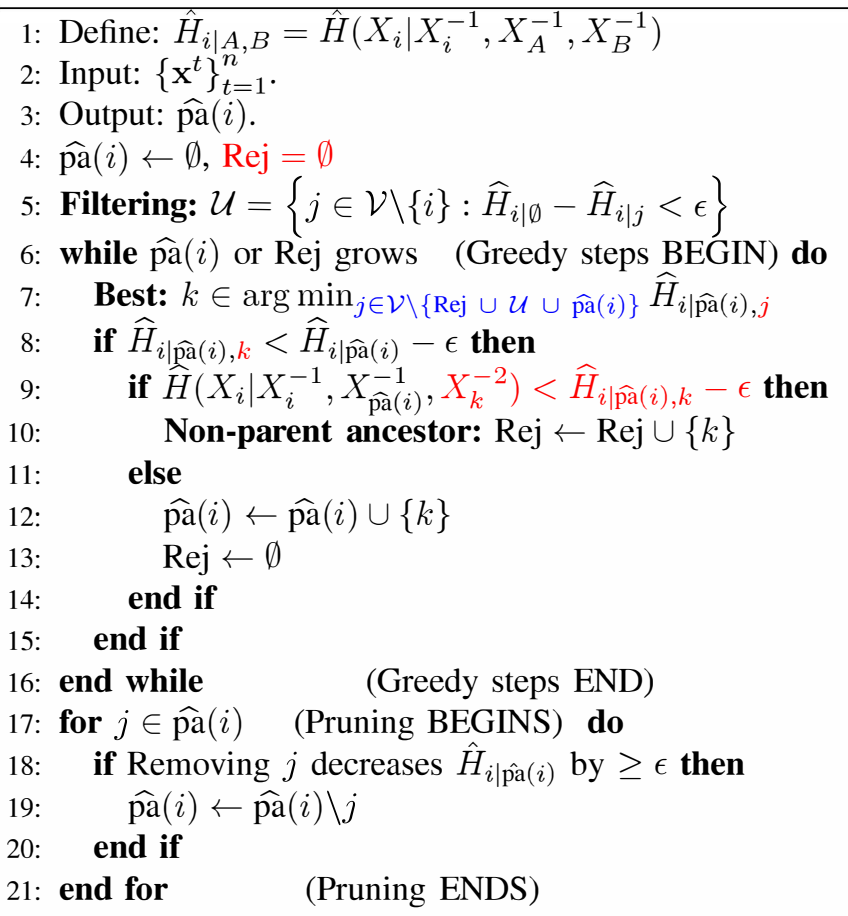

Fig. 3: Description of the greedy algorithm

nodes can be picked and rejected as non-parents ancestor before a node is picked as a parent or the algorithm stops. This takes $O\left(p^{2}\right)$ worst case computational steps to find one parent. There may be at most $p$ parents and hence the worst case computational complexity is $O\left(p^{3}\right)$ per node.

\section{MAIN RESUlts}

In what follows we present the theoretical guarantees for the proposed algorithm. We show that our algorithm can provably recover the underlying causal relationships in a multi-dimensional Markov process with high probability. Moreover we show that the number of samples required by this algorithm for learning the causal relationship with high probability grows logarithmically with the dimension of the multivariate process.

\section{A. Assumptions}

To seek provable learning guarantees on the proposed greedy algorithm, the process of interest $\mathbf{X}$ has to follow some structure. Next, we discuss the structural properties that are required to be satisfied and subsequently discuss their significance.

Assumption 1: The underlying undirected graph corresponding to the directed graph that captures causal relationships in the multi-dimensional process is degree-bounded by $\eta$ and all transitions in the joint process (1) that occur with positive probability are bounded below by a constant $\lambda>0$.

Assumption 2: For all $A \subseteq \mathrm{pa}(i), j \in \mathrm{pa}(i) \backslash A$, and $S \subseteq$ $\mathcal{V}$ such that there exist no directed path between $S$ and $i$ (i.e. 


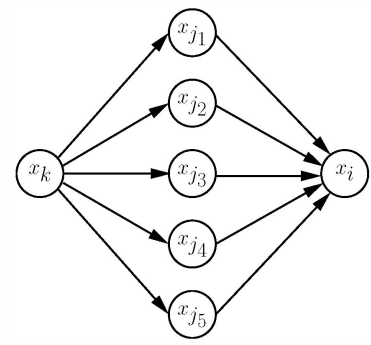

(a) Illustrating the influential ancestor effect.

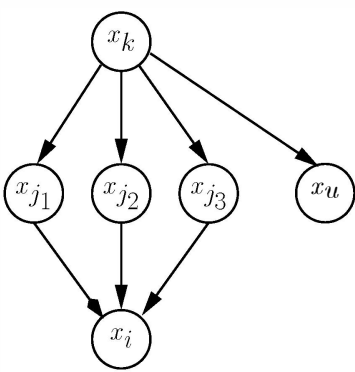

(b) Illustrating the case with strong effect of non-ancestor process

Fig. 2
$S$ does not contain any ancestor of $i$ ), we have

$$
\begin{array}{r}
H\left(X_{i} \mid X_{i}^{-1}, X_{A}^{-1}, X_{S}^{-1}\right)- \\
H\left(X_{i} \mid X_{i}^{-1}, X_{A}^{-1}, X_{S}^{-1}, X_{j}^{-1}\right)>2 \epsilon
\end{array}
$$

Assumption 3: For all $B \subseteq \mathcal{V} \backslash\{i\}$ and $k \in R \backslash$ pa(i) (there is a directed path from $\forall r \in R$ to $i$ ), we have

$$
H\left(X_{i} \mid X_{i}^{-1}, X_{B}^{-1}, X_{k}^{-1}\right)-H\left(X_{i} \mid X_{i}^{-1}, X_{B}^{-1}, X_{k}^{-2}\right)>2 \epsilon .
$$

Assumption 4: For $i, j \in \mathcal{V}$, if there does not exist a directed path from $j$ to $i$, then

$$
H\left(X_{i} \mid X_{i}^{-1}\right)-H\left(X_{i} \mid X_{i}^{-1}, X_{j}^{-1}\right)<2 \epsilon
$$

if the undirected distance $\tilde{d}(i, j)$ between node $i$ and node $j$ in the underlying graph, i.e., length of the shortest path from $i$ and $j$ after removing directions from edges is at least $d$, an integer independent of the graph size.

Assumption 1 ensures that the graph has degree $\mathrm{O}(1)$ and all possible transitions happens often enough to be estimated correctly. This is a standard assumptions in literature on sample based graph learning.

By the definition of causality and causal graph (as in Sec. II), inequality 8 is always true for any $j \in \mathrm{pa}(i), A \subseteq \mathrm{pa} \backslash j$ and $S \subset \mathcal{V} \backslash j$, for $\epsilon=0$. As every node has a finite parent set and every parent has non-zero influence (otherwise it is not a parent), for some $\epsilon>0$ the strict inequality is true for $S=\emptyset$. Hence in Assumption 2 we only impose the additional condition that the strict inequality is also true for $S$ containing non-ancestor nodes which is likely when each parent has significant influence on its child.

Note that $H\left(X_{i} \mid X_{i}^{-1}, X_{B}^{-1}, X_{k}^{-1}, X_{k}^{-2}\right)=$ $H\left(X_{i} \mid X_{i}^{-1}, X_{B}^{-1}, X_{k}^{-2}\right)$ for a non-parent ancestor $k$ of $i$. This is due to the Markov property in time and the fact that effect of a non-parent ancestor reaches in more than one time step and $X_{i}^{t}$ depends on $X_{k}^{t-1}$ only through value of $X_{k}$ at time prior to $t-1$. Also note that as conditioning reduces entropy, $H\left(X_{i} \mid X_{i}^{-1}, X_{B}^{-1}, X_{k}^{-1}, X_{k}^{-2}\right) \leq H\left(X_{i} \mid X_{i}^{-1}, X_{B}^{-1}, X_{k}^{-1}\right)$. Assumption 3 only requires that this inequality be strict. Note that as the graph is finite and there are only finite such inequalities there exists an $\epsilon>0$ to bound the gap in the strict inequality from below.
Assumption 4 is related to the correlation decay assumption, which is also prevalent in most graph learning problems. This assumption however is weaker than the standard correlation decay assumption as it requires that influence of a process associated with a sufficiently far away node is below a threshold only if it is a non-ancestral node.

In all the cases $2 \epsilon$ is picked for the ease of exposition in the proofs. We require $\epsilon>0$ because we have to estimate entropies from samples. Hence a non-zero margin is needed to avoid errors in decision making based on these values. We show in our theoretical guarantees that the requirement on the margin $(\epsilon)$ goes to zero with increasing number of samples.

\section{B. Theoretical Guarantees}

Our algorithm has provable guarantees on the probability of correct recovery of the true graph and on the sample complexity. Our main theoretical result is summarized in the following theorem which involves the mixing time of the Markov chain. Note that $\gamma$ mixing time of a Markov chain with stationary distribution $\pi(\cdot)$ is defined as $\min \{t$ : $\left.\forall A,\left|\mathbb{P}\left(X_{t} \in A\right)-\pi(A)\right|<\gamma\right\}$.

Theorem V.1. $\mathcal{G}$ be the causal directed graph associated with a p-variate stationary Markovian time series $\mathbf{X}$ on a finite space as defined in (1) and $\mathbf{X}$ satisfies the assumptions $1-4$ with $T$ being its 0.1 -mixing time. Given $\epsilon=2 \tilde{\epsilon} \log \frac{|\mathcal{X}|}{\tilde{\epsilon}}$, if number of samples $n=\Omega\left(\frac{T}{\lambda \tilde{\epsilon}^{2}} \ln \left(\frac{p^{\left(\eta^{d}+\eta+1\right)}|\mathcal{X}|^{\left(\eta^{d}+\eta+1\right)}}{\delta}\right)\right)$, the proposed greedy algorithm recovers the true underlying causal directed graph with probability at least $1-\delta$. The computational complexity of the algorithm is $O\left(p^{4}\right)$.

The theorem implies that with high probability the greedy algorithm correctly learns the underlying causal relationship in the multi-dimensional time-series and the number of samples required for this is logarithmic in the dimension of the process. Note that though in the order sense the sample complexity is similar to that of the greedy learning of undirected graphical model, this algorithm requires more samples. The reason behind this is that Markovian samples being strongly correlated have lesser rate of information than that of i.i.d samples. Also as one needs to exploit the information hidden in spread of influence over time to 
learn the bad case (diamond graph), the sample complexity increases. Proof of this theorem bases on using the properties of the underlying directed causal graph of the process and on a concentration result for Markov chains. Here we briefly describe the main intuition behind the proof. Details of the proof are presented in section VII.

As addition of a parent node into the conditioning set strictly reduces the conditional directed entropy of a process, the estimated parent set constructed by the algorithm during the greedy steps includes the true parent set, with high probability. The algorithm also ensures that no non-parent ancestor is included in the estimated parent set by checking the two step conditional entropy. Finally the pruning method ensures that no node which does not have any direct effect is excluded. Guarantees on sample complexity follow using concentration results for Markov chains, and noting that the true parent set and the parent set estimated by the greedy algorithm are $O(1)$.

Remark V.2. The algorithm can be easily extended to block Markov processes using the same basic ideas. All proofs also generalize to the block Markov case when similar assumptions as in Sec. V-A are used for such processes.

\section{Simulations}

We evaluate the performance of our algorithm on computer generated data. Given a graph, for each node $i$, we use a scalar $\nu_{i}$ and a vector $\theta_{\cdot i}$ to capture influences of past value of itself and that of other processes. For each node $i$ and $x_{i}^{t} \in\{0,1\}$, we generate a kernel (as in (1)) with $p\left(x_{i}^{t} \mid x_{i}^{t-1}, x_{\mathrm{pa}(i)}^{t-1}\right) \propto$ $\exp \left(\nu_{i} x_{i}^{t} x_{i}^{t-1}+\sum_{j \in \mathrm{pa}(i)} \theta_{j i} x_{i}^{t} x_{j}^{t-1}\right)$. We consider three types of directed graphs; tree (only solid edges), class 1 graphs where we put long loops at the end of a tree and class 2 graphs where for each directed (solid) edge $(i, j)$ of the tree we put another edge $(j, i)$ (see Fig. 4a). For a fixed graph, we consider two cases, 1) $\nu_{i} \in[0.2,1]$ and $\theta_{j i} \in[0.5,1]$, and 2) $\nu_{i} \in[0.5,1]$ and $\theta_{j i} \in[0.5,1]$, where these coefficients are chosen uniformly at random. In all the plots we present probability of successful (no extra or missing edge) recovery at different sample sizes.

Fig. 4b presents performance of our algorithm with parameters as in case 1 for class 1 and class 2 graphs. Note that class 2 graphs are harder to learn compared to the class 1 graphs due to the presence of strong circular dependency. A point to note from the plot is that the sample complexity grows slowly with number of nodes as stated in the Theorem V.1.

To compare the performance of our algorithm with a benchmark we choose directed mutual information based Chow-Liu type algorithm proposed specifically for trees in [17]. This algorithm that learns only directed trees is asymptotically optimal if the process is stationary. In Fig. 4c we present comparisons of our algorithm with that of [17]. As observed Chow-Liu performs better in terms of sample complexity as it is restricted to trees where pairwise statistics are sufficient to learn the graph. On contrary an algorithm designed for a general graph has to estimate statistics (directed conditional entropy in our case) involving at least three processes. As the sample complexity to estimate statistics is proportional to the number of variables (nodes), requirement of 1.5 times samples compared to Chow-Liu is unavoidable for an algorithm for general graphs.

Next to compare on general graphs we use the well known logistic regression proposed in [2] for Ising models. Note that the simulated process is not i.i.d Ising model but the transition probability is equivalent to an Ising function. Thus we can use logistic regression to learn this model if we consider the process $\left(\mathbf{X}_{t-1}, \mathbf{X}_{t}\right)$ and for each $i$ consider two separate nodes $i$ and $i^{-}$for processes $\left\{X_{i}(t)\right\}$ and $\left\{X_{i^{-}}(t)=X_{i}(t-1)\right\}$ respectively. Fig. $4 \mathrm{~d}$ is obtained after optimally tuning of regression parameters. As logistic regression bases strongly on independence between samples this does not extend well to processes even when the kernel is Ising-like. For general Markovian multivariate processes our algorithm would be more useful in many applications.

\section{PROOF OF THEOREM V.1}

Note that our algorithm is based on directed conditional entropy estimates computed using empirical probability distribution. Next, we quantify the difference between these empirical estimates and their true value, i.e. estimation error, in terms of number of samples available to the algorithm.

Lemma VII.1. Given number of samples satisfies $n=$ $\Omega\left(\frac{T}{\lambda \tilde{\epsilon}^{2}} \ln \left(\frac{p^{D+1}|\mathcal{X}|^{D+1}}{\delta}\right)\right)$, we have with probability at least $1-\delta$,

$$
\begin{aligned}
& \left\|p\left(X_{i}, X_{A}^{-1}\right)-\hat{p}\left(X_{i}, X_{A}^{-1}\right)\right\|_{1} \leq \tilde{\epsilon} \\
& \forall i \in[p] \text { and } A \subseteq[p] \text { s.t. }|A| \leq D
\end{aligned}
$$

Before we prove Lemma VII.1, we state a Chernoff style concentration bound for Markov Chains due to [25], which help us prove Lemma VII.1. The following theorem states that the probability that the empirical mean of a function of state space of a Markov chain is far from its expected value decays exponentially with the number of samples used to calculate empirical mean. Here the expectation of the function is over the invariant distribution of the underlying Markov chain. We state the theorem in slightly weaker form that is sufficient for our purpose. For more details on the following result, readers may refer to [25].

Theorem VII.2. Concentration result for Markov chains: Consider a Markov chain $M$ with $\gamma$-mixing time $T_{\gamma}(\gamma<$ 1/8). Let $Y_{1}, \ldots, Y_{n}$ be $n$ step random walk on $M$. Define $Z=\sum_{t=1}^{n} f\left(Y_{t}\right)$, where $f(\cdot): \mathcal{Y} \rightarrow \mathbb{R}$ be a function from state space of $M$ to real line. Let $\mu$ denote the expectation of $f(\cdot)$ with respect to invariant distribution of $M$. Then for $\tilde{\epsilon} \in[0,1]$ we have

$$
\mathbb{P}\left[\left|\frac{Z}{n}-\mu\right| \geq \tilde{\epsilon} \mu\right] \leq 2 C\left(\pi_{1}\right) \exp \left(-\tilde{\epsilon}^{2} \mu n /\left(72 T_{\gamma}\right)\right)
$$

Proof: (Lemma VII.1) Let $\mathrm{x}^{t}=\left(x_{1}^{t}, x_{2}^{t}, \ldots, x_{p}^{t}\right)$ denote the values that $p$-variate time-series take at time $t$. We 


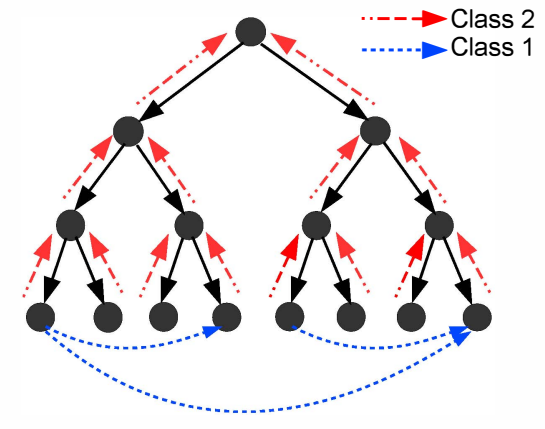

(a) Two classes of graphs considered in simulations.

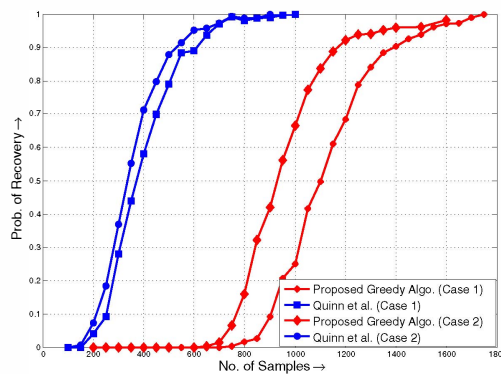

(c) Comparison with tree learning algorithm due to [17] for 31 node binary tree.

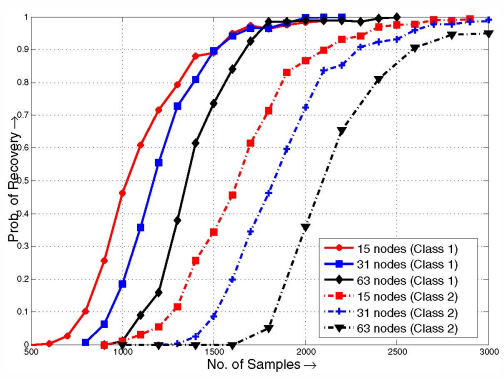

(b) Performance of the proposed algorithm with case 1 parameters $\left(\nu_{i} \in[0.2,1]\right.$ and $\left.\theta_{j i} \in[0.5,1]\right)$.

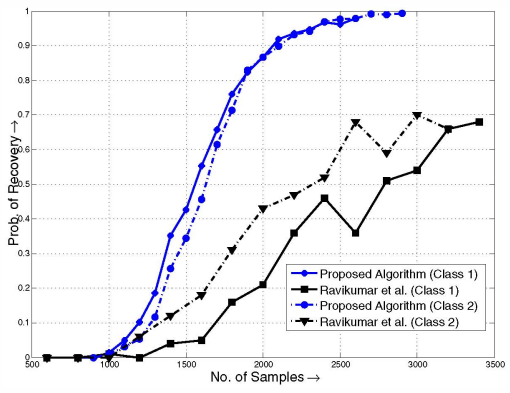

(d) Comparison with logistic regression based graph learning algorithm due to [2].

Fig. 4 know that evolution of this vector process is Markovian with transition probability defined in (1). Next, we construct an $n-2$ length Markov chain $M$ with its state at $t$ is denoted by $\mathrm{y}^{t}=\left(\mathrm{x}^{t-2}, \mathrm{x}^{t-1}, \mathrm{x}^{t}\right)$. The transition matrix of this new Markov chain $M$ is determined by $P$, the transition matrix of the original Markov chain over $\mathrm{x}^{t}$. Let $f_{\mathbf{y}_{S}}(\mathbf{Y})=\mathbf{1}\left\{\mathbf{Y}_{S}=\mathbf{y}_{S}\right\}$ be an indicator function defined over the realization of Markov chain $M$, which takes value 1 if and only if the components of a random vector $\mathrm{Y}$ indexed by a set $S \subseteq[3 p]$ take value $\mathbf{y}_{S}$. Since we are interested in estimating quantities of the form $p\left(X_{i}^{t}=x_{i}, X_{A}^{t-1}=\right.$ $\left.x_{A}, X_{k}^{t-2}=x_{k}\right)$, we pick $S$ to be a subset of $[3 p]$ such that $\mathrm{Y}_{S}=\left(X_{i}^{t}, X_{A}^{t-1}, X_{k}^{t-2}\right)$. Note that by stationarity $p\left(X_{i}^{t}=x_{i}, X_{A}^{t-1}=x_{A}, X_{k}^{t-2}=x_{k}\right)$ is same for all $t$ and we call it $p\left(x_{i}, x_{A}^{-1}, x_{k}^{-2}\right)$ Here $i \in[p]$ and $A \subseteq[p]$. The empirical estimate of $p\left(x_{i}, x_{A}^{-1}, x_{k}^{-2}\right)$ is defined as follows

$\hat{p}\left(x_{i}, x_{A}^{-1}, x_{k}^{-2}\right)=\frac{1}{n-2} \sum_{t=3}^{n} \mathbf{1}\left\{\mathbf{Y}_{S}^{t}=\left(x_{i}, x_{A}^{-1}, x_{k}^{-2}\right)\right\}$

Note that

$$
\mathbb{E}\left[\hat{p}\left(x_{i}, x_{A}^{-1}, x_{k}^{-2}\right)\right]=p\left(x_{i}, x_{A}^{-1}, x_{k}^{-2}\right)
$$

Next, applying Theorem VII. 2 we get

$$
\begin{array}{r}
\mathbb{P}\left[\left|\hat{p}\left(x_{i}, x_{A}^{-1}, x_{k}^{-2}\right)-p\left(x_{i}, x_{A}^{-1}, x_{k}^{-2}\right)\right| \geq \tilde{\epsilon} p\left(x_{i}, x_{A}^{-1}, x_{k}^{-2}\right)\right] \\
\leq 2 C\left(\pi_{1}\right) \exp \left(\frac{-\tilde{\epsilon}^{2} p\left(x_{i}, x_{A}^{-1}, x_{k}^{-2}\right)(n-2)}{72 T_{\gamma}}\right)
\end{array}
$$

where $T_{\gamma}$ is the $\gamma$-mixing time for the Markov chain $\mathbf{Y}$ which is same as mixing time for $\mathbf{X}$.
When we restrict ourself to only bounded conditioning sets, i.e. sets $A$ s.t. $|A| \leq D-1$. Note that $D$ is the bound on the conditioning set $A \cup k$.

$$
\begin{array}{r}
\mathbb{P}\left[\left\|\hat{p}\left(x_{i}, x_{A}^{-1}, x_{k}^{-2}\right)-p\left(x_{i}, x_{A}^{-1}, x_{k}^{-2}\right)\right\|_{1} \geq \tilde{\epsilon}\right] \leq \\
p\left(\begin{array}{c}
p \\
D
\end{array}\right)|\mathcal{X}|^{D+1} 2 C\left(\pi_{1}\right) \exp \left(\frac{-\tilde{\epsilon}^{2} \lambda(n-2)}{72 T_{\gamma}}\right)
\end{array}
$$

where $\lambda>0$ is a uniform lower bound on $p\left(x_{i}, x_{A}^{-1}, x_{k}^{-2}\right)$ for all $\left(x_{i}, x_{A}^{-1}, x_{k}^{-2}\right) \in \mathcal{X}^{|A|+2}$. For the choice of $n$ specified in the statement of Lemma VII.1, the quantity in right hand side in (12) is bounded by $\delta$.

Note that $\widehat{H}()=H(\widehat{p})$. By choosing $\tilde{\epsilon}$ suitably we can show that empirical entropy and true entropy values are $\frac{\epsilon}{2}$ close and this follows using the following inequality [24].

$$
\|H(p)-H(\widehat{p})\|_{1} \leq-\|p-\widehat{p}\|_{1} \log \frac{\|P-\widehat{P}\|_{1}}{|\mathcal{X}|}
$$

Given $\epsilon=2 \tilde{\epsilon} \log \frac{|\mathcal{X}|}{\tilde{\epsilon}}$ this inequality implies that

$$
H-H^{\prime}-\epsilon<\widehat{H}-\widehat{H^{\prime}}<H-H^{\prime}+\epsilon
$$

for any two entropy quantities $H$ and $H^{\prime}$ computed based on $A$ as mentioned above. This results in the particular choice of $\tilde{\epsilon}$ in Theorem V.1.

In what follows, the rest of the ingredients of proof of Theorem V.1 are presented in the following order. First we prove that steps $4-16$ of the algorithm output a parent set 
estimate for each node, which contains the true parent set and does not contain any ancestor node. Then we show that size of this parent set estimate is $\mathrm{O}(1)$ and pruning recovers the true parent set with high probability. Since the learnt parent set is $\mathrm{O}(1)$, pruning step has polynomial computational complexity in $p$. We prove the main theorem using the following two lemmas which are true at the same time with probability at least $1-\delta$ by the lemma VII.1.

Lemma VII.3. For $n$ in Lemma VII.1 with high probability, for each $i \in \mathcal{V}$ we have that the parent set learnt at the end of step 16, $\widetilde{p a}(i)$, contains pa $(i)$, and for any node $j \in$ $\widetilde{p a}(i) \backslash p a(i)$, there does not exist a directed path from $j$ to $i$. Moreover, we have that $|\tilde{p a}(i)|<\eta^{d}+\eta$.

Proof: For this proof we only need to consider the steps 5-16 that we call the greedy part of the algorithm. By inequality 14, under assumption 3 step 9 ensures that no non-parent ancestor node that has a directed path to node $i$ is included in $\widehat{\mathrm{pa}}(i)$. Similarly by assumption 4 and inequality 14 , the algorithm does not include any non-ancestral node which is more than $d$ distance (in $\tilde{d}(\cdot, \cdot)$ as defined in Sec. $\mathrm{V}$-A) away from node $a$. Therefore, the parent set estimate $\widetilde{\mathrm{pa}}(i)$ at the end of the step 16 contains only parents and non-ancestor nodes that are within $d$ distance. Since the underlying undirected graph is degree bounded by $\eta$, size of the set $\widetilde{\text { pa }}(i)$ is at most $\eta^{d}+\eta$. Also note that as the size of the conditioning set at any step of the greedy algorithm is bounded by the size of the estimated parent set plus one, substituting $D=\eta^{d}+\eta$ in Lemma VII.1 shows that sample complexity for a greedy step is of the same order as in Lemma VII.1.

Note that $\widehat{p a}(i)$ never includes a non-parent ancestor node. Hence using inequality 14, it follows from assumption 2 that at any stage of the algorithm adding a true parent node to the estimated parent set reduces directed conditional entropy by more than $\epsilon$. Therefore, the greedy part never fails to include a true parent.

Lemma VII.4. For $n$ in Lemma VII.1, for each node $i \in \mathcal{V}$ the pruning process recovers the true parent set with high probability.

Proof: As the algorithm removes one node from the conditioning set at each pruning step, size of the conditioning set is upper bounded by $\eta^{d}+\eta$. Thus, the sample complexity result follows.

By inequality 14 and assumption 2, removing a true parent node from the estimated parent set always increases estimated directed conditional entropy by more than $\epsilon$. Thus a true parent node is never dropped during a pruning step. By lemma VII.3, $\widetilde{\text { pa }}(i)$ contains $\mathrm{pa}(i)$. Hence the inequality 14 along with Markov property of the underlying directed graph imply that removing any non-parent node increases estimated directed conditional entropy by an amount strictly less than $\epsilon$. Hence any non-parent node is removed during pruning process.

Complexity result follows similarly as in [1]. Note that by
Lemma VII.1, the statements of Lemmas VII.3 and VII.4 are true at the same time with high probability $1-\delta$. Theorem V.1 follows by combining these lemmas.

\section{REFERENCES}

[1] P. Netrapalli, S. Banerjee, S. Sanghavi, and S. Shakkottai. Greedy learning of markov network structure. In Allerton, pages 613-636, 2011.

[2] P. Ravikumar, M. J. Wainwright, and J. D. Lafferty. High-dimensional graphical model selection using 11-regularized logistic regression. Annals of Statistics, 2010.

[3] P. Abbeel, D. Koller, and A. Y. Ng. Learning factor graphs in polynomial time and sample complexity. JMLR, 2006.

[4] G. Bresler, E. Mossel, and A. Sly. Reconstruction of markov random fields from samples: Some observations and algorithms. In APPROX/RANDOM, 2008.

[5] C. I. Chow and C. N. Liu. Approximating discrete probability distributions with dependence tre. IEEE Transactions on Information Theory, 1968.

[6] R. Dahlhaus. Graphical interaction models for multivariate time series. Metrika, 51(2), 2000.

[7] F. R. Bach and M. I. Jordan. Learning graphical models for stationary time series. IEEE Transaction on Signal Processing, 2004.

[8] A. Jalai and S. Sanghavi. Learning the dependence graph of time series with latent factors. In ICML, 2012.

[9] Ursula Gather, Michael Imhoff, and Roland Fried. Graphical models for multivariate time series from intensive care monitoring. Statistics in Medicine, 2002.

[10] C. J. Quinn, T. P. Coleman, N. Kiyavash, and N. G. Hatsopoulos. Estimating the directed information to infer causal relationships in ensemble neural spike train recordings. J Comput Neurosci, 2010.

[11] Raymond Salvador, John Suckling, Christian Schwarzbauer, and Ed Bullmore1. Undirected graphs of frequency-dependent functional connectivity in whole brain networks. Phil. Trans. of R. Soc. B, 360, 2005.

[12] V. Y. F. Tan and A. Wilsky. Sample complexity for topology estimation in networks of lti systems. In 18th IFAC World Congress, Italy, 2011.

[13] I. L. MacDonald and W. Zucchini. Hidden Markov Models and Other models for discrete valued time series. Chapman and Hall/CRC, 1997.

[14] N. D. Mukhopadhyay and S. Chatterjee. Causality and pathway search in microarray time series experiment. Bioinformatics, 2007.

[15] S. Kirshner, P. Smyth, and A. W. Robertson. Conditional chowliu tree structures for modeling discrete-valued vector time series. In Proceedings of the 20th conference on Uncertainty in artificial intelligence, 2004.

[16] A. Anandkumar, V. Y. F. Tan, and A. Wilsky. High-dimensional structure estimation in ising models: Local separation criterion. Annals of Statistics, 40, 2012.

[17] C. Quinn, T. P. Coleman, and N. Kiyavash. Causal dependence tree approximations of joint distributions for multiple random processes. submitted to IEEE Transactions on Information Theory, 2011.

[18] J. L. Massey. Causality, feedback and directed information. In Proc. Intl. Symp. Inf. Th. Appl., 1990.

[19] Pierre-Olivier Amblard and Olivier J. J. Michel. The relation between granger causality and directed information theory: A review. Entropy, 15(1), 2013.

[20] Jiantao Jiao, Haim H. Permuter, Lei Zhao, Young-Han Kim, and Tsachy Weissman. Universal estimation of directed information. CoRR, abs/1201.2334, 2012.

[21] N. Friedman, K. Murphy, and S. Russell. Learning the structure of dynamic probabilistic networks. In UAI, 1998.

[22] J. F. Blimes. Dynamic bayesian multinets. In UAI, 2000

[23] H. Lahdesmaki and I. Shmulevic. Learning the structure of dynamic bayesian networks from time series and steady state measurements. Machine Learning, 71, 2008.

[24] T. M. Cover and J. A. Thomas. Elements of information theory. WileyInterscience, New York, NY, USA, 1991.

[25] K. Chung, H. Lam, Z. Liu, and M. Mitzenmacher. Chernoff-hoeffding bounds for markov chains: Generalized and simplified. In STACS 2012, 2012. 